\title{
Three-dimensional bioprinted liver tissue for transplantation: hope or hype?
}

\author{
Feihu Xie $^{1}$, Yao Xiao ${ }^{2}$, Minshan Chen ${ }^{1}$ \\ ${ }^{1}$ Department of Hepatobiliary Surgery, Sun Yat-sen University Cancer Center, Guangzhou, China; ${ }^{2}$ Peking Union Medical College, Beijing, China \\ Correspondence to: Prof. Chen Minshan. Department of Hepatobiliary Surgery, Sun Yat-sen University Cancer Center, 651 Dongfeng Road East, \\ Guangzhou, China. Email: chenmsh@sysucc.org.cn.
}

Submitted Jun 24, 2020. Accepted for publication Jul 03, 2020.

doi: $10.21037 / \mathrm{hbsn}-20-549$

View this article at: http://dx.doi.org/10.21037/hbsn-20-549

Liver transplantation is one of the best available choices for those with end-stage liver diseases and liver cancer who meet Milan criteria $(1,2)$. However, a critical shortage of available liver donors is the main problem associated with transplantation, which presumably cannot be solved in the foreseeable future. Various effects have been made in the last few decades to compensate for this shortage, including developing different kinds of artificial liver support systems (3). Unfortunately, it is still far away from meeting the demand.

Recently, there are achievements in three-dimensional (3D) bioprinting of organs with multiple functions in vitro that might lighten up the hope of future implantable artificial organs, including the liver. We believe the closest try could be a recent fascinating work by Yang et al. published in the journal "Gut" with the title of "three-dimensional bioprinted hepatorganoids prolong survival of mice with liver failure" (4). The authors described a new liver tissue model constructed by $3 \mathrm{D}$ bioprinting of HepaRG cells. They give this $3 \mathrm{D}$ printed liver a special name of "Hepatorganoids" (3 DP-HOs). Further, $3 \mathrm{DP}-\mathrm{HO}$ was actually transplanted into animal models with liver failure (5), which significantly prolonged the survival. This study is, to the best of our knowledge, the current most interesting and far front research in this field. There are at least several intriguing establishments and findings on this 3DPHOs. First, HepaRG cells were managed to differentiate into hepatocytes with the full ability of regeneration in the study. After 7 days of dimethyl sulfoxide (DMSO) induction, HepaRG cells in the 3DP-HOs frame could stably differentiate into hepatocytes, and in the mean time, carried out almost all the liver functions. The liver function-related proteins, such as albumin, multidrugresistance-associated protein 2, and CYP3A4, are markedly higher in 3DP-HOs than that in 2D cultures. The cells with $3 \mathrm{D}$ structure showed the capability of accumulating glycogen, uptaking and releasing indocyanine green, which are unique features of the normal liver cells. Though the inherent mechanism is not clear at the moment, it surely deserves further in-depth investigations in this field. Second, as the most important implication of the study, 3DP-HOs manifests as human liver functional units inside the abdomen of mice. After the transplantation of 3DPHOs into the abdominal cavities of $\mathrm{F} / \mathrm{R}$ mice, which represent a well-established liver failure model (5), the mice acquired human-specific drug metabolism activities and can respond to certain inducers, such as sodium phenobarbital, and showed increased activities of CYP1A2 or CYP3A4 at the levels that are comparable to those of primary human hepatocytes. Exposing F/R mice transplanted with 3DP-HOs to debrisoquine, the production of humanspecific metabolite 4-hydroxydebrisoquine (4-OH-DEB) is significantly elevated. This is a clear message that $3 \mathrm{DP}$ HOs manifested as human liver functional units after being transplanted into mice. Third, a very interesting finding of this study is the vascularization growing into 3DP-HOs after transplantation. 3DP-HOs transplants started becoming neovascularized at day 14 after the transplantation, which can be clearly visualized 4 weeks after the transplantation. Last but not least, 3DP-HOs transplants prolonged the survival duration of the liver 
failed mice. After the transplantation, several indicators for the impaired liver functions, such as the serum levels of alanine aminotransferase, total bilirubin, and gammaglutamyl transpeptidase, etc., are dramatically decreased, while the indicators for regular liver function, such as the serum levels of albumin, total protein, and prealbumin are significantly increased. F/R mice that received 3DP-HOs transplants have significantly longer survival and less weight loss. The results suggest that the $3 \mathrm{DP}-\mathrm{HO}$ transplants may alleviate the liver injury process and compensate for impaired liver function. Now, the question is whether these findings are strong or sufficient enough to bring any hope or hint in future artificial organ transplantation.

Let's return to the topic, i.e., is $3 \mathrm{D}$ bioprinted liver tissue for future transplantation a hope or just a hype? On the bright side, there is no doubt that $3 \mathrm{DP}-\mathrm{HOs}$ managed to show liver functions both in vitro and in vivo; it survived, grew, and vascularized in a liver failure model. It could serve as a positive model for further studies. However, this study raised more questions than it answered. First of all, the present 3DP-HOs were constructed with HepaRG cells, which were originally derived from a liver tumor (6), although it has almost no tumor characteristics after regenerations. Of course, the present study is only a demonstration indicating that something could be possible to achieve. We presume that the very next step for this transplantation purpose could be $3 \mathrm{D}$ bioprinting of human primary liver cells, which has been reported very recently, but still not mature yet. The structure and function of the liver are most sophisticated and complex among human organs. A simple and straight forward issue comes out right the way is the bile secretion, which is a unique and indispensable function of the liver. The reported 3DP-HOs surely failed to complete the complicated bile production, secretion, duct drainage system, and not even reached close to that. Moreover, the strength of the present 3DP-HOs structure is highly dependent on the mixture of gelatin and sodium alginate solution. It is a fragile structure with barely acceptable strength for short-term transplantation in vivo, thus the long-term stability of the structure is not optimistic. More importantly, due to the absence of endothelial cells in 3DP-HOs model, real vascularization did not actually occur in this model.

The liver is the largest and one of the most complicated organs in the human body. To date, the liver cannot be truly remodeled in vitro by any type of technologies. However, it should really be encouraged to make the upmost efforts with new advanced science and techniques to mimic the morphology and biological activities of the liver. The miracle happens in our history. One would never imagine from 19 century that organs could be practically and routinely transplanted in the clinic in the future. As an emerging technology, 3D bioprinting could be one of the possible options, which characterized by superb delicacy and feasibility in reconstructing desired tissues or organs, when compared with organoids and other bio-artificial liver systems (7). The establishment of 3DP-HOs in this study is the first demonstration that $3 \mathrm{D}$ bioprinted liver tissues possess the capability to serve as transplantable grafts in mice with liver failure and compensate for impaired liver functions. It is a progress forward in the field of artificial liver, and it might become a possibility to substitute for missing donors or bridge the process for liver transplantation in the future. Until now, however, it should not be over interpreted for its implications.

On the other hand, the utility of $3 \mathrm{D}$ cell bioprinting technique could be expanded from fabricating complex organs to establishing in vitro models for various cancers. The process of $3 \mathrm{D}$ bioprinting is adjusted by rewritable computer programs, which endows it with the flexibility to construct cancer models in different styles according to specific needs. The bioprinted models also share identical structures and contents, including cell density and distribution. This kind of uniformity, which is absent in the current cancer models like organoids and patient-derived xenografts (PDX), is essential to performing drug screening for cancers. If one keeps imagination unlocked, there is always a way ahead.

\section{Acknowledgments}

Funding: None.

\section{Footnote}

Provenance and Peer Review: This article was commissioned by the editorial office, Hepatobiliary Surgery and Nutrition. The article did not undergo external peer review.

Conflicts of Interest: All authors have completed the ICMJE uniform disclosure form (available at http://dx.doi. org/10.21037/hbsn-20-549). The authors have no conflicts of interest to declare.

Ethical Statement: The authors are accountable for all aspects of the work in ensuring that questions related 
to the accuracy or integrity of any part of the work are appropriately investigated and resolved.

Open Access Statement: This is an Open Access article distributed in accordance with the Creative Commons Attribution-NonCommercial-NoDerivs 4.0 International License (CC BY-NC-ND 4.0), which permits the noncommercial replication and distribution of the article with the strict proviso that no changes or edits are made and the original work is properly cited (including links to both the formal publication through the relevant DOI and the license). See: https://creativecommons.org/licenses/by-nc-nd/4.0/.

\section{References}

1. Kim D, Cornman-Homonoff J, Madoff DC. Preparing for liver surgery with "Alphabet Soup": PVE, ALPPS, TAE-PVE, LVD and RL. Hepatobiliary Surg Nutr 2020;9:136-51.

2. Sahara K, Farooq SA, Tsilimigras DI, et al. Immunotherapy utilization for hepatobiliary cancer

Cite this article as: Xie F, Xiao Y, Chen M. Three-dimensional bioprinted liver tissue for transplantation: hope or hype? HepatoBiliary Surg Nutr 2020;9(6):788-790. doi: 10.21037/hbsn20-549 in the United States: disparities among patients with different socioeconomic status. Hepatobiliary Surg Nutr 2020;9:13-24.

3. Yang L, Wu T, Li J, et al. Artificial liver treatment improves survival in patients with hepatitis B virus-related acute-on-chronic liver failure: a case-control matched analysis. Hepatol Res 2020;50:656-70.

4. Yang H, Sun L, Pang Y, et al. Three-dimensional bioprinted hepatorganoids prolong survival of mice with liver failure. Gut 2020. [Epub ahead of print].

5. Grompe M, al-Dhalimy $M$, Finegold $M$, et al. Loss of fumarylacetoacetate hydrolase is responsible for the neonatal hepatic dysfunction phenotype of lethal albino mice. Genes Dev 1993;7:2298-307.

6. Gripon P, Rumin S, Urban S, et al. Infection of a human hepatoma cell line by hepatitis B virus. Proc Natl Acad Sci U S A 2002;99:15655-60.

7. Mota C, Camarero-Espinosa S, Baker MB, et al. Bioprinting: from tissue and organ development to in vitro models. Chem Rev 2020;120:10547-607. 